\title{
INTRAOPERATIVE BREAKAGE OF A REUSED 25 GAUGE ILM PEELING FÓRCEPS A CASE REPORT
}

\author{
Madhurima Roy, Sangeeta Roy, Aniruddha Maiti, Nishita Yadav \\ Susrut Eye Foundation and Research Centre, Kolkata
}

\begin{abstract}
Financial constraints have forced developing countries to reuse instruments in retinal surgeries, which may cause unintentional problems sometimes. We describe a rare case of inadvertent intraoperative breakage of a reused 25-gauge ILM forceps. A 59 years male presented with painless diminution of vision in the right eye for 4 months. Fundus examination revealed an epiretinal membrane with central macular thickness of $747 \mu \mathrm{m}$. He underwent pars plana vitrectomy. Intraoperatively after internal limiting membrane peeling, during removal of the pieces of the membrane, one blade of ILM peeling forceps broke apart. The blade had been successfully removed and the patient recovered uneventfully.

Keywords: 25 gauge ILM peeling forceps, forceps breakage, instrument reuse, pars plana vitrectomy Cite This Article: ROY, Madhurima et al. Intraoperative fracture of 25 guage ILM peeling forceps. International Journal of Retina, [S.I.], v. 4, n. 2, p. 178, sep. 2021. ISSN 2614-8536. Available at: $<$ https://www.ijretina.com/index.php/ijretina/article/view/166>.doi: https://doi.org/10.35479/ijretina.2021.v ol004.iss002.166.
\end{abstract}

\section{Correspondence to:}

Madhurima Roy,

Susrut Eye Foundation and Research

Centre, Kolkata

kutunandini@yahoo.com

\section{BACKGROUND}

Newer small gauge vitrectomy instruments have made vitreo-retina surgeries more efficient and less time-consuming with faster visual recovery. Grieshaber DSP ILM forceps allow to work on fine membranes with precise grasping, but the forceps are made disposable after a single-use. To our knowledge, this is probably the first report of accidental intraoperative breakage of a reused ILM peeling forceps among 25 gauge instruments.

\section{CASE PRESENTATION}

A 59-year-old male presented with painless diminution of vision in the right eye (RE) for the last 4 months. He had no systemic co-morbidities. His bestcorrected visual acuity (BCVA) was 20/100 in RE and 20/30 in the left eye (LE). Anterior segment examination of both eyes was unremarkable. Fundus examination of RE revealed epiretinal membrane (ERM) causing puckering of retinal layers and LE fundus was normal. Optical coherence tomography (OCT) of RE showed thick ERM and retinal edema with central retinal thickness of 747 $\mu \mathrm{m}$ [Fig. 2a]. He underwent 25 gauge, 3ports pars plana vitrectomy surgery. Triamcinolone-assisted posterior vitreous detachment (PVD) was induced. Brilliant blue-green dye 0.05\% (BBG- Ocublue Plus, Aurolab) was used to stain the internal limiting membrane (ILM). With the help of negative staining, peeling of ERM was done over macula by Grieshaber DSP End-Grasping ILM 25- gauge forceps (Alcon Laboratories, Inc:)[Fig. 1a]. 
Repeat BBG staining was performed and 2 disc diameters ILM peeling over macula was completed. During ILM peeling, manipulation was done with the endoilluminator pipe and ILM forceps to remove the stuck pieces of ILM, which led to accidental breakage of one blade of the forceps from the spring and fell onto the posterior pole [Fig. 1b, 1c]. One bleeding vessel during peeling had been cauterized. There was no iatrogenic break. The broken blade was then grasped with Grieshaber DSP MAX grip forceps
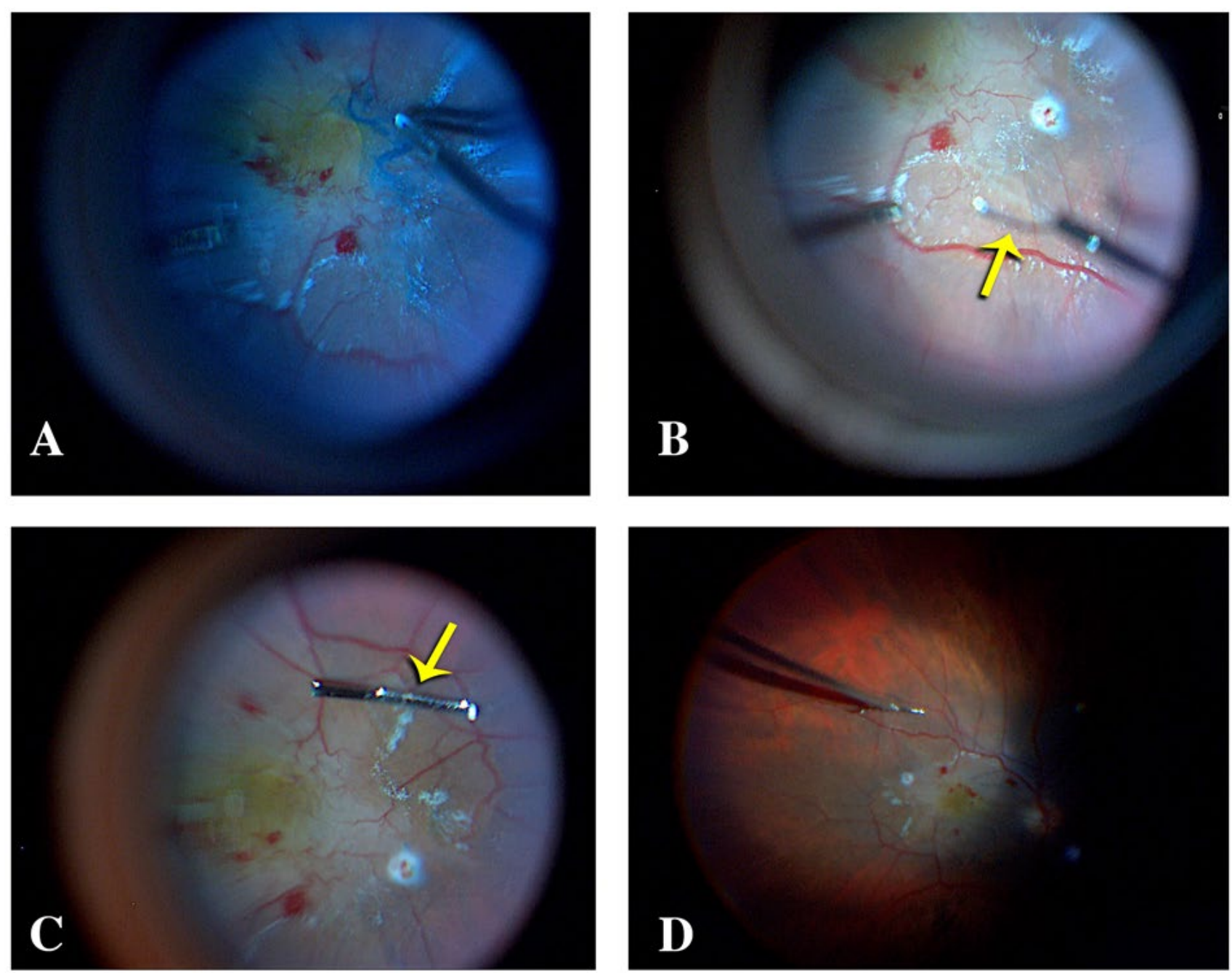

Figure. 1A: ERM peeling with the ILM forceps. 1B: ILM forceps showing one blade and another blade accidentally broke down (yellow arrow). 1C: The broken blade is seen over the retina without causing any iatrogenic tear or bleeding. 1D: Retrieval of the broken tip with the help of MAXgrip forceps. 

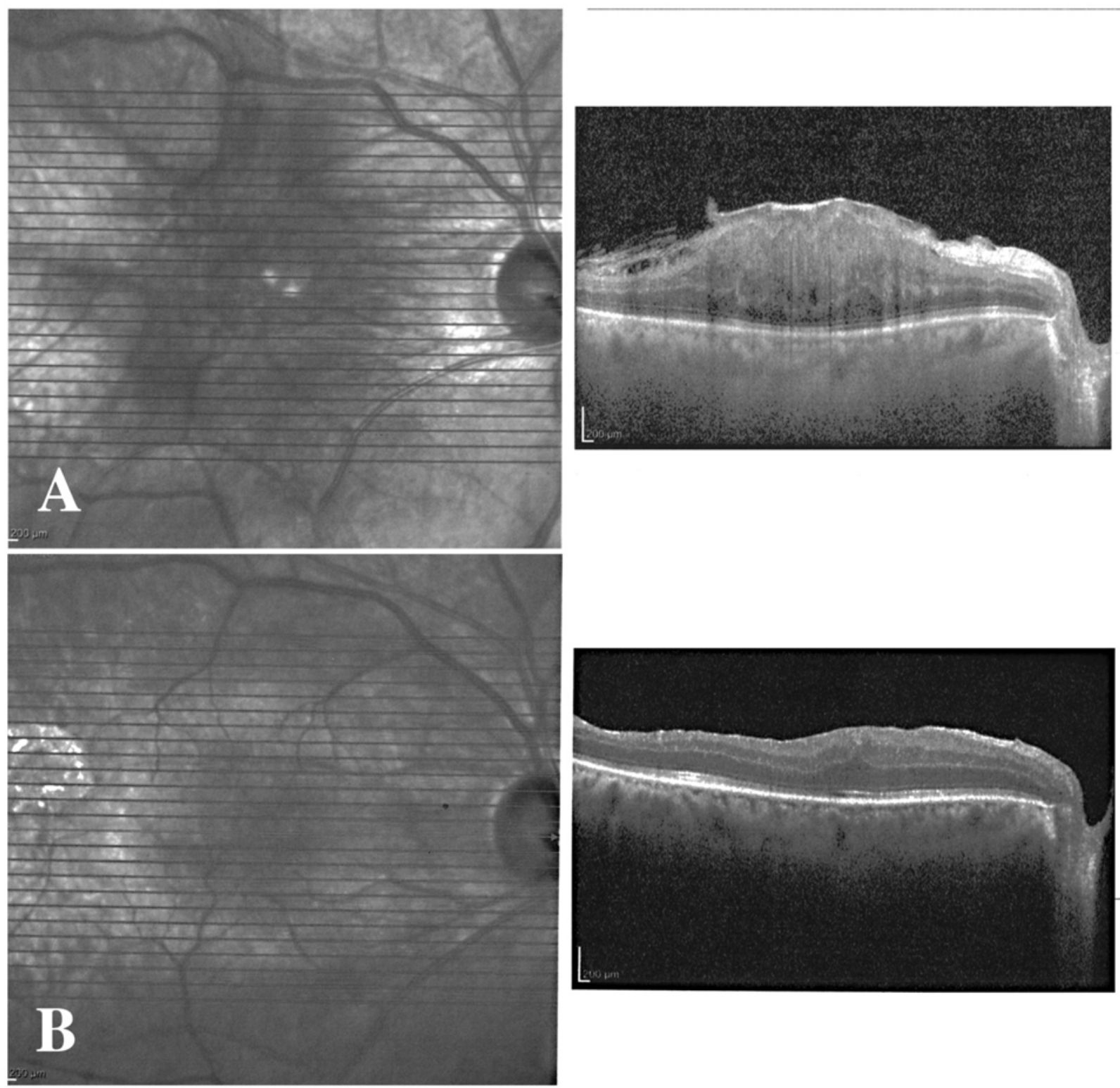

Figure 2A. Preoperative OCT showing hyper-reflective ERM causing distortion of all retinal layers and edema. Fig. 2B: 6-weeks-postoperative OCT after ERM removal, showing reduction of retinal edema to $413 \mu \mathrm{m}$.

\section{DISCUSSION}

With the advent of small gauge vitrectomy, vitreo-retina surgeries have become more efficient with shorter operating times and faster visual recovery than their earlier counterpart. Preferably, these are of single-use, disposable instruments. High volume institutions like ours where most of the patients are self-paying and resource constraint being one of the major factors, it is often not possible to use disposable ones for each case, therefore most of us reuse these instruments to make them economically viable for our patients. These high-quality 25 gauge instruments can stand up to limited reuse with no problem. In this case, the ILM forced fracture that occurred during ERM peeling, was presumed to be due to loss of spring action because of repeated use after ethylene-oxide (ETO) sterilization. Intra-operatively, ILM forceps was properly handled and was well functioning till the blade fell off. 
The faulty instrument was rechecked by the manufacturer company Alcon Inc. and found corrosion at the level of spring of the forceps.

To the best of our knowledge, this is the only report of such an incident among 25- gauge ILM peeling instruments. Akira Hirata reported a case of $20 \mathrm{G}$ kryptonite forceps fracture during ILM peeling in diabetic macular edema . ${ }^{1}$ Also, there are case series of three patients having breakage of kryptonite forceps due to crack at the shaft during vitreoretinal surgery. ${ }^{2}$ Furthermore, few cases have been reported on accidental dislocation of silicon sleeves of 20 gauge extrusion cannula during vitrectomy. ${ }^{3,4} \mathrm{We}$ use ethylene oxide sterilization routinely for fine ophthalmic instruments at our institution. Not surprisingly, there are some concerns about the repeated reuse of these instruments. Repeated usage after ETO sterilization, can result in surface damage leading to metal corrosion or fatigue and durability issues. Another safety concern with the reusable instruments is the potential for crosscontamination. Although a 13-year review of 12989 vitrectomy cases with reused instruments showed a $0.10 \%$ incidence of endophthalmitis, which is comparable with the endophthalmitis rate using disposable instruments in previous literature. ${ }^{5}$ In our case postoperative period was uneventful.

\section{CONCLUSION}

While reusing the instruments all these factors like sterility, cost and fragility should be considered. One must be certain that reused instruments are always sharp and not bent or damaged during proper sterilization. Both visual and functional examination under the microscope should be done before intraocular use and multiple uses of these disposable micro-instruments should be avoided. The Key is to strike a balance in the cost-benefit ratio.

\section{REFERENCES}

1. Hirata A. Fracture of intraocular forceps during vitrectomy. Acta Ophthalmologica Scandinavica. 2007 Mar;85(2):223

2. Bartz-Schmidt KU, El-Araj I, Gelisken F, Reinthal E, Ziemssen $\mathrm{F}$, Eter $\mathrm{N}$ et al. Intraoperative breakage of 20 -gauge Tano forceps. American journal of ophthalmology. 2006 Jan 1;141(1):215-7.

3. Ascaso FJ, Arias L, Caminal J, Herrera L, Del Buey MA, Cristóbal J. Two cases of accidental dislocation of the silicone sleeve of an extrusion cannula into the vitreous cavity. Case reports in ophthalmology. 2012;3(3):438-42.

4. Agrawal S, Gupta SC, Agrawal J, Agrawal TP. Silicone sleeve of extrusion cannula as an intraocular foreign body. Indian journal of ophthalmology. 2002 Mar 1;50(1):58-9.

5. Silpa-Archa S, Kumsiang K, Preble JM. Endophthalmitis after pars plana vitrectomy with reused single-use devices: a 13-year retrospective study. International Journal of Retina and Vitreous. $2021 \operatorname{Dec}_{i} 7(1): 1-9$.

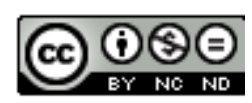

This work licensed under Creative Commons Attribution 